\title{
ARTICLE
}

\section{The hypoglycemic mechanism of catalpol involves increased AMPK-mediated mitochondrial biogenesis}

\author{
Deng-qiu Xu ${ }^{1}$, Chun-jie $\mathrm{Li}^{1}$, Zhen-zhou Jiang ${ }^{1,4}$, Lu Wang ${ }^{1}$, Hong-fei Huang ${ }^{6}$, Zhi-jian Li ${ }^{1,5}$, Li-xin Sun ${ }^{1,4}$, Si-si Fan ${ }^{1}$, \\ Lu-yong Zhang ${ }^{1,2,4}$ and Tao Wang ${ }^{1,3}$
}

Mitochondria serve as sensors of energy regulation and glucose levels, which are impaired by diabetes progression. Catalpol is an iridoid glycoside that exerts a hypoglycemic effect by improving mitochondrial function, but the underlying mechanism has not been fully elucidated. In the current study we explored the effects of catalpol on mitochondrial function in $d b / d b$ mice and C2C12 myotubes in vitro. After oral administration of catalpol $\left(200 \mathrm{mg} \cdot \mathrm{kg}^{-1} \cdot \mathrm{d}^{-1}\right)$ for 8 weeks, $d b / \mathrm{db}$ mice exhibited a decreased fasting blood glucose level and restored mitochondrial function in skeletal muscle. Catalpol increased mitochondrial biogenesis, evidenced by significant elevations in the number of mitochondria, mitochondrial DNA levels, and the expression of three genes associated with mitochondrial biogenesis: peroxisome proliferator-activated receptor gammaco-activator 1 (PGC-1a), mitochondrial transcription factor A (TFAM) and nuclear respiratory factor 1 (NRF1). In C2C12 myotubes, catalpol significantly increased glucose uptake and ATP production. These effects depended on activation of AMP-activated protein kinase (AMPK)-mediated mitochondrial biogenesis. Thus, catalpol improves skeletal muscle mitochondrial function by activating AMPK-mediated mitochondrial biogenesis. These findings may guide the development of a new therapeutic approach for type 2 diabetes.

Keywords: catalpol; AMPK; mitochondria; biogenesis; type 2 diabetes; skeletal muscle

Acta Pharmacologica Sinica (2020) 41:791-799; https://doi.org/10.1038/s41401-019-0345-2

\section{INTRODUCTION}

Diabetes mellitus (DM) is a serious endocrine and metabolic disease. The vast majority of DM cases fall into two broad etiopathogenetic categories [1]. Type 1 diabetes mellitus (T1DM) accounts for only $5 \%-10 \%$ of all DM cases and is caused by $\beta$-cell destruction, usually leading to complete insulin deficiency. Type 2 DM (T2DM) accounts for $90 \%-95 \%$ of all DM cases and is caused by insulin resistance (IR), sometimes accompanied by defects in insulin secretion. Most anti-diabetic drugs exert unwanted side effects (hypoglycemia and gastrointestinal problems) [2]. There is an urgent need to develop safer and more effective drugs to treat T2DM and insulin resistance.

Changes in cell energy metabolism and nutrient partitioning are responsible for the development of insulin resistance [3]. Mitochondria generate most of the cell's energy via aerobic respiration and are indispensable for life [4]. Mitochondrial dysfunction may affect energy production, thereby impairing cell function, and has been implicated in many acute and chronic diseases [5-7]. A strong association of insulin resistance and T2DM development with mitochondrial dysfunction is evident, and mitochondrial dysfunction causes insulin resistance [8-10]. However, the details of this association remain unclear. Some studies have shown that lifestyle interventions (exercise or caloric restriction) increase insulin sensitivity, delaying or preventing diabetes onset, and insulin sensitivity is closely related to increases in mitochondrial mass and oxidative phosphorylation [11, 12]. A defect in oxidative phosphorylation caused by a reduced number of mitochondria may affect fatty acid metabolism, ultimately causing insulin resistance $[11,13]$. In addition, one study found that a reduction in mitochondrial content was caused by a reduced type I:Il muscle fiber ratio [13]. A few studies on diabetics have reported substantial decreases in mitochondrial volume and obvious abnormalities in mitochondrial morphology, consistent with the mitochondrial ultrastructural changes and decreased oxidative phosphorylation activity evident in T2DM patients $[14,15]$. Together, these data suggest that the mitochondrion may be an important therapeutic target for the treatment of T2DM.

Mitochondrial biogenesis is dynamically regulated; new mitochondria are constantly produced to maintain the appropriate number of morphologically normal active organelles to meet the constantly changing bioenergetic needs of the cell via oxidative metabolism [16]. Therefore, compounds that stimulate mitochondrial biogenesis may improve mitochondrial dysfunction. Mitochondrial biogenesis is coordinately controlled by transcription factors, nuclear hormone receptors, and transcriptional coactivators. Peroxisome proliferator-activated receptor gamma coactivator 1, a member of the PGC-1 family of transcriptional coactivators, is the master regulator of mitochondrial biogenesis

\footnotetext{
${ }^{1}$ Jiangsu Key Laboratory of Drug Screening, China Pharmaceutical University, Nanjing 210009, China; ${ }^{2}$ Center for Drug Research and Development, Guangdong Pharmaceutical University, Guangzhou 510006, China; ${ }^{3}$ Jiangsu Center for Pharmacodynamics Research and Evaluation, China Pharmaceutical University, Nanjing 210009 , China; ${ }^{4}$ Key Laboratory of Drug Quality Control and Pharmacovigilance, China Pharmaceutical University, Nanjing 210009, China; ${ }^{5}$ Department of Toxicology Laboratory, Xinjiang Institute of Traditional Uyghur Medicine, Xinjiang Laboratory of Uyghur Medical Prescription, Urumqi 830049, China and ${ }^{6}$ School of Pharmacy, China Pharmaceutical University, Nanjing 211198, China Correspondence: Lu-yong Zhang (lyzhang@cpu.edu.cn) or Tao Wang (wangtao1331@cpu.edu.cn)
}

Received: 25 August 2019 Accepted: 2 December 2019

Published online: 14 January 2020 
in many tissues [17]. PGC-1a controls the expression levels of mitochondrial genes and induces mitochondrial biogenesis by interacting with transcription factors such as nuclear respiratory factors (NRFs), peroxisome proliferator-activated receptors, and estrogen receptor-related receptors (ERRs) [18]. Mitochondrial transcription factor A, a DNA-binding stimulatory protein, regulates mitochondrial DNA transcription and replication to maintain the mitochondrial DNA (mtDNA) content [19]. PGC-1a interacts with NRF1 to activate mitochondrial transcription factor A (TFAM) expression, thereby stimulating mitochondrial biogenesis [20]. Furthermore, T2DM development is associated with decreased PGC-1a expression in the liver and skeletal muscle caused by a reduction in PGC-1a-dependent gene expression. This compromises mitochondrial biogenesis and further impairs mitochondrial ATP synthesis [21]. Overall, mitochondrial biogenesis regulated by PGC-1a may be critical for T2DM treatment.

Catalpol is an iridoid glucoside of Rehmannia glutinosa that exerts significant hypoglycemic effects on different diabetic models $[22,23]$. A recent study indicated that orally administered catalpol has beneficial effects on insulin resistance thus lowering blood glucose [24]. We recently showed that catalpol improved HFD/STZ-induced muscle mitochondrial dysfunction [25]. Insulin resistance and mitochondrial dysfunction are closely related. Moreover, it is still debated whether catalpol exerts direct mitochondrial protection or insulin-sensitizing effects on skeletal muscles. Although the hypoglycemic effect of catalpol is related to increased mitochondrial function, the detailed molecular mechanism remains unknown. Here, we explored how catalpol improves mitochondrial function in $d b / d b$ mice and $\mathrm{C} 2 \mathrm{C} 12$ myotubes.

\section{MATERIALS AND METHODS}

Materials and reagents

Catalpol (purity $>99 \%$ ) was obtained from Nanjing Spring \& Autumn Biological Engineering Co., Ltd. (Nanjing, China). Fetal bovine serum was purchased from ExCell Biotech (Cat No: FSP500, Shanghai, China). Fatty acid-free bovine serum albumin (BSA) was obtained from Merck (Kenilworth, NJ, USA). Mito Tracker Red (Molecular Probes) and 2-[N-(7-nitrobenz-2-oxa-1,3-diazol-4-yl) amino]-2-deoxy-D-glucose (2-NBDG) were purchased from Invitrogen (Carlsbad, CA, USA). Other reagents were purchased from Sigma-Aldrich (St. Louis, MO, USA) unless otherwise stated.

\section{Animal studies}

All experimental procedures were conducted in accordance with institutional guidelines for the care and use of laboratory animals in China. Male C57BL KS/J- $d b / d b$ mice (8-9 weeks old, $40-45 \mathrm{~g}$ in body weight, $n=16$ animals) and their normoglycemic littermates (8-9 weeks old, $18-20 \mathrm{~g}$ in body weight, $n=8$ animals) were obtained from the Nanjing University Animal Model Research Center (Nanjing, China). Sixteen C57BLKS/J-db/ $d b$ mice were randomly allocated to the $d b / d b$ mouse group or the $200 \mathrm{mg} / \mathrm{kg}$ catalpol-treated group (orally administered for 8 consecutive weeks). All animals were housed in an airconditioned room maintained at a temperature of $20-23^{\circ} \mathrm{C}$ with a 12/12-h light/dark cycle and were provided with standard mouse chow and water ad libitum. At the end of the experimental period, all mice were anesthetized using isoflurane following $12 \mathrm{~h}$ of fasting. Blood was collected from the inferior vena cava for the determination of glucose, $\mathrm{HbA} 1 \mathrm{c}$, triglyceride (TG), total cholesterol (TC), high-density lipoprotein cholesterol (HDL-C) and low-density lipoprotein cholesterol (LDL-C) levels. Then, the mice were killed, and tibialis anterior (TA) muscle sections were collected. The TA muscle sections were used for Western blotting, Rt-qPCR and histological analysis. All dissected tissues were immediately frozen in liquid nitrogen and stored at $-80^{\circ} \mathrm{C}$ until analysis.

\begin{tabular}{|c|c|c|c|}
\hline Gene & & & $\mathrm{NCBI}$ \\
\hline \multirow[t]{2}{*}{ PGC-1 $\alpha$} & Forward & TATGGAGTGACATAGAGTGTGCT & NC_000071.6 \\
\hline & Reverse & CCACTTCAATCCACCCAGAAAG & \\
\hline \multirow[t]{2}{*}{ TFAM } & Forward & TGATTGGCACCGATCCTCG & NC_000076.6 \\
\hline & Reverse & CCACAGCGTCATATCATCCAG & \\
\hline \multirow[t]{2}{*}{ ERR- $\alpha$} & Forward & GACGGCAGAAGTACAAACGG & NC_000085.6 \\
\hline & Reverse & CAACCAGCAGATGCGACAC & \\
\hline \multirow[t]{2}{*}{ Nrf1 } & Forward & AGCACGGAGTGACCCAAAC & NC_000072.6 \\
\hline & Reverse & TGTACGTGGCTACATGGACCT & \\
\hline \multirow[t]{2}{*}{$\beta$-Actin } & Forward & AGGTGTGGTGCCAGATCTTC & NC_000071.6 \\
\hline & Reverse & GGACTCCTATGTGGGTGACG & \\
\hline \multirow[t]{2}{*}{ ND1 } & Forward & CAGCCTGACCCATAGCCATAATAT & NC_005089.1 \\
\hline & Reverse & TGATTCTCCTTCTGTCAGGTCGAA & \\
\hline
\end{tabular}

Glucose and insulin tolerance tests

The glucose tolerance test $(2.0 \mathrm{~g} / \mathrm{kg}$ i.p.) was performed in the animals following $6 \mathrm{~h}$ of fasting. Serum glucose concentrations were detected by using a glucometer (Bayer Corporation, Mishawaka, IN, USA) at 0, 30, 60, 90, and 120 min after glucose injection. The insulin tolerance test $(0.75 \mathrm{lU} / \mathrm{kg}$ i.p.) was performed in the animals following $4 \mathrm{~h}$ fasting. Serum glucose concentrations were determined using a glucometer at 0, 30, 60, and 120 min after insulin injection. The areas under the curves (AUCs) of the insulin tolerance test results were calculated [10].

Mitochondrial assays

Mitochondria were separated from fresh TA muscles as previously described [25]. The relative mitochondrial DNA content was measured by quantitative polymerase chain reaction (PCR) as described previously [26]. Nuclear $\beta$-actin and ND1 were used to represent nDNA and mtDNA. The primer pairs used for PCR are listed in Table 1.

\section{Determination of mitochondrial membrane potential and ATP} content

The mitochondrial membrane potential $(\Delta \psi \mathrm{m})$ in the TA muscles of $d b / d b$ mice was evaluated using a JC- 1 mitochondrial membrane potential detection kit, as previously described [26]. The ATP content of the isolated mitochondria was determined using a Cell Titer-Glo ${ }^{\circledR} 2.0$ Assay Kit (Promega, Madison, WI, USA) according to the manufacturer's instructions.

\section{Electron microscopy}

Fresh TA muscles were immediately fixed with cold $2.5 \%$ glutaraldehyde in phosphate-buffered saline (PBS, $\mathrm{pH}=7.4$ ). All samples were processed, as previously described [10]. Images were acquired using a JEM-1010 electron microscope (Jeol USA Inc., Peabody, MA, USA). For each group, the number of mitochondria was assayed from 24 images from 8 animals using Image-Pro Plus software (Media Cybernetics, Rockville, MD, USA).

\section{Cell culture}

$\mathrm{C} 2 \mathrm{C} 12$ cells were purchased from the American Tissue Culture Collection (ATCC) and grown in DMEM growth medium (Dulbecco's modified Eagle's medium supplemented with $10 \%$ fetal bovine serum and $1 \%$ penicillin/streptomycin) in a $5 \% \mathrm{CO}_{2}$ incubator at $37^{\circ} \mathrm{C}$ as previously described [27]. Confluent $\mathrm{C} 2 \mathrm{C} 12$ myoblasts were differentiated into myotubes by switching the medium to medium containing $2 \%$ horse serum and $1 \%$ penicillin/ streptomycin when the cells reached $90 \%-100 \%$ confluency. Differentiated myotubes were serum-starved overnight and then 
incubated in serum-free media containing 10,30 , or $100 \mu \mathrm{M}$ catalpol or $1 \mathrm{mM}$ metformin. Stimulated myotubes were collected for Western blotting or real-time PCR. Differentiated myotubes were incubated in serum-free medium containing $2 \mu \mathrm{M}$ compound $\mathrm{C}$ for $2 \mathrm{~h}$ and then incubated in new serum-free medium containing 10,30 , or $100 \mu \mathrm{M}$ catalpol or $1 \mathrm{mM}$ metformin for $24 \mathrm{~h}$ as described in our previous report [22].

Glucose uptake activity measurement using 2-NBDG

Glucose uptake activity in myotubes was measured using 2-NBDG, as described previously [28]. Briefly, myotubes were washed with cold PBS and incubated in glucose-free DMEM with $60 \mu \mathrm{M}$ 2-NBDG for $1 \mathrm{~h}$. Then, the myotubes were washed twice with cold PBS to remove free 2-NBDG. Finally, the myotubes were suspended in cold PBS and transferred to the wells of a 96-well fluorescence microplate.

\section{Mitochondrial staining}

Following incubation with $50 \mathrm{mM}$ D-glucose for $24 \mathrm{~h}$ and treatment with catalpol, cells were stained with $100 \mathrm{nM}$ MitoTracker Red for $30 \mathrm{~min}$ at $37^{\circ} \mathrm{C}$, as described in our previous report [22]. Mitochondria were visualized under a confocal microscope, and the images were analyzed using Image-Pro Plus software (Media Cybernetics, Rockville, MD, USA).

Real-time quantitative polymerase chain reaction (PCR)

Total RNA was extracted from the TA muscle using TRIzol reagent and an RNeasy Kit (Vazyme Biotech, Nanjing, China) according to the manufacturer's instructions. Complementary DNA (cDNA) was synthesized from total RNA using a cDNA Synthesis Kit (Vazyme Biotech, Nanjing, China). The resulting cDNAs were amplified using a SYBR Green Master Mix Kit in an ${ }^{\mathrm{iQ}^{\mathrm{T}} \mathrm{M}} 5$ real-time $\mathrm{PCR}$ detection system (Bio-Rad Laboratories, Hercules, CA, USA), as reported previously [29]. Gene expression was evaluated via the $\Delta \Delta C T$ method using glyceraldehyde 3-phosphate dehydrogenase (GAPDH) as a reference gene. The primer pairs used for PCR are listed in Table 1.

Western blot analysis

Excised TA muscles were dissolved in total protein extraction buffer (keyGEN Biotech, Nanjing, China) with protease and phosphatase inhibitors. The total protein $(30 \mu \mathrm{g})$ was fractionated on $6-12 \%$ sodium dodecyl sulfate (SDS) polyacrylamide gels and transferred to nitrocellulose membranes (Millipore, Billerica, MA, USA) by electroblotting. The membranes were blocked in Tris-buffered saline containing $5 \%$ milk and then incubated overnight at $4{ }^{\circ} \mathrm{C}$ with one of the following primary antibodies: rabbit anti-TFAM (Cat No: ab131607, Abcam, Cambridge, MA, USA), rabbit anti-PGC-1a (Cat No: 19142-1, Abcam), rabbit anti-PFKP (Cat No: 8164T, CST, Danvers, MA, USA), rabbit anti-phosphor-ACC (Cat No: 3661s, CST), rabbit anti-ACC (Cat No: 3662s, CST), rabbit anti-COX 1 (Cat No: A6564, ABclonal, Wuhan, Hubei, China), rabbit anti-NDUFS3 (Cat No: A8013, ABclonal), rabbit anti-SDHA (Cat No: A2594, ABclonal), rabbit anti-ATP5A1 (Cat No: A5884, ABclonal), rabbit anti-UQCRC2 (Cat No: A4184, ABclonal), mouse anti-GAPDH (Cat No: sc-32233, Santa Cruz, Felton, CA, USA) or mouse anti- $\beta$-actin (Cat No: sc-69879, Santa Cruz).

Immunohistochemistry

For immunohistochemistry, TA muscle samples were fixed in $4 \%$ paraformaldehyde (PFA), and paraffin-embedded sections were incubated overnight at $4{ }^{\circ} \mathrm{C}$ with a primary antibody against myosin heavy chain II (MY-32) (1:100; ab51263, Abcam, Cambridge, MA, USA) [30]. Stained tissues were examined under a light microscope (BX53, Olympus, Japan).

Statistical analysis

All obtained data were analyzed with one-way analysis of variance (ANOVA) followed by Tukey's multiple comparison posttest using
Table 2. Blood biochemical parameters.

\begin{tabular}{|c|c|c|c|}
\hline & $\mathrm{CON}$ & $d b / d b$ & Catalpol \\
\hline Glucose $(\mathrm{mmol} / \mathrm{L})$ & $5.3 \pm 1.5$ & $29.1 \pm 3.7^{* * *}$ & $20.2 \pm 4.4^{* \#}$ \\
\hline Body weight (g) & $28.5 \pm 1.2$ & $47.1 \pm 3.2^{* * *}$ & $48.3 \pm 2.9^{* * *}$ \\
\hline HbA1c (\%) & $3.29 \pm 0.63$ & $6.64 \pm 0.65^{*}$ & $4.2 \pm 0.43^{\#}$ \\
\hline $\mathrm{TG}(\mathrm{mmol} / \mathrm{L})$ & $1.17 \pm 0.19$ & $1.66 \pm 0.15^{*}$ & $1.41 \pm 0.12$ \\
\hline TC (mmol/L) & $0.73 \pm 0.05$ & $1.13 \pm 0.2^{*}$ & $0.78 \pm 0.11^{\#}$ \\
\hline $\mathrm{HDL}-\mathrm{C}(\mathrm{mmol} / \mathrm{L})$ & $1.15 \pm 0.21$ & $0.75 \pm 0.18^{* *}$ & $1.02 \pm 0.33$ \\
\hline LDL-C (mmol/L) & $1.33 \pm 0.29$ & $2.87 \pm 0.38^{* *}$ & $1.21 \pm 0.44^{\#}$ \\
\hline \multicolumn{4}{|c|}{$\begin{array}{l}\text { Data are means } \pm \mathrm{SD} \text {, with } n=8 \text { per group. }{ }^{*} P<0.05,{ }^{* *} P<0.01,{ }^{* * *} P<0.001 \\
\text { compared with controls; }{ }^{\#} P<0.05 \text { vs. } d b / d b . C O N \text { control mice, } d b / d b d b / d b \\
\text { mice, Catalpol db/db mice treated with Catalpol, TG triglyceride, TC } \\
\text { cholesterol, } H D L-C \text { high-density lipoprotein cholesterol, LDL-C low-density } \\
\text { lipoprotein cholesterol }\end{array}$} \\
\hline
\end{tabular}

GraphPad 6.0 (Graph-pad Software Inc., San Diego, CA, USA). Data are presented as the mean \pm SD. Values with $P<0.05$ were considered statistically significant.

\section{RESULTS}

Catalpol lowers fasting blood glucose levels and ameliorates mitochondrial dysfunction in the skeletal muscle of $d b / d b$ mice Body weight and the serum levels of glucose, $\mathrm{HbA} 1 \mathrm{C}, \mathrm{TG}, \mathrm{TC}$, and LDL-C were increased in the $d b / d b$ mice compared with the control mice, whereas, the serum level of HDL-C was decreased in the $d b / d b$ mice. Catalpol treatment decreased the serum levels of glucose, HbA1c, TC, and LDL-C (Table 2). Moreover, catalpol treatment increased insulin sensitivity (Fig. 1a, b) but had no effect on glucose tolerance (Fig. 1c). As we reported previously, catalpol improved skeletal muscle mitochondrial function [25]. The mitochondrial membrane potential of the skeletal muscle of $d b / d b$ mice was $60 \%$ lower than that of control mice but increased after catalpol treatment (Fig. 1d). As glucose utilization and mitochondrial activity are associated with changes in ATP production [31], we performed an ATP assay to evaluate mitochondrial function. The catalpol treatment of $d b / d b$ mice increased ATP production (Fig. 1e). Although the mtDNA copy number was only slightly reduced in the skeletal muscle of $d b / d b$ mice, catalpol markedly increased the mtDNA level (Fig. 1f). To explore whether catalpol increases the number of mitochondria in mouse skeletal muscle, we assessed both mitochondrion numbers and morphology via electron microscopy. The number of mitochondria was lower in $d b / d b$ mice than in control mice. After the catalpol treatment of $d b / d b$ mice, the mitochondrion number increased, but mitochondrial morphology was abnormal. The skeletal muscles of the $d b / d b$ mice contained abundant lipid droplets, reflecting ectopic lipid deposition (Fig. 1g, h). Mitochondrial dynamics are usually associated with quality control and the functional maintenance of bioenergetics [32]. The expression levels of three genes associated with mitochondrial biogenesis (PGC-1a, NRF1, and TFAM) were markedly decreased in the skeletal muscle of $d b / d b$ mice, but these decreases were reversed by catalpol (Fig. 1i), as revealed by Western blotting (Fig. 1j, k).

Catalpol ameliorates the abnormal muscle fiber ratio in $d b / d b$ mice

Recent evidence has shown that a shift in the muscle fiber type from oxidative type I to the more glycolytic type II damages the muscles of obese subjects with impaired glucose tolerance [33]. We quantified the muscle fiber types by immunohistochemical staining of TA muscle sections and assessed the findings. The proportion of myosin heavy chain I (MHC I) fibers was decreased, 

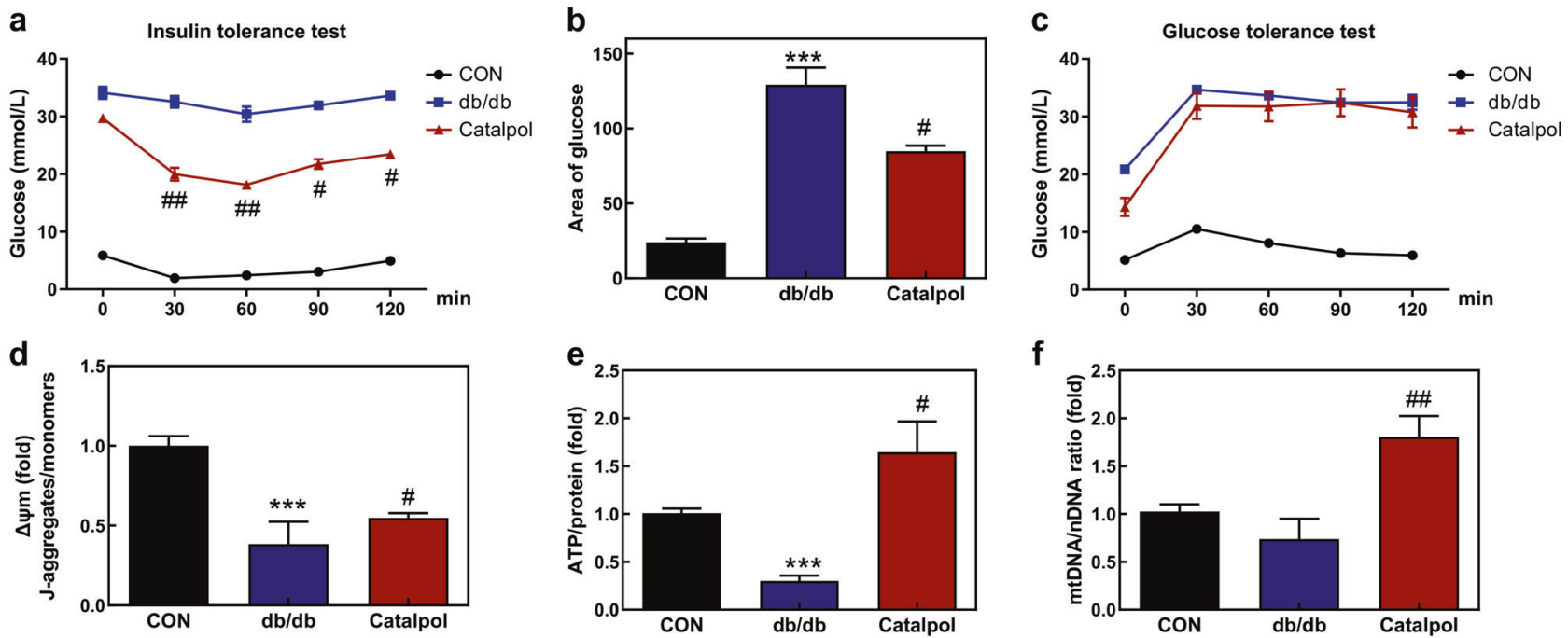

f

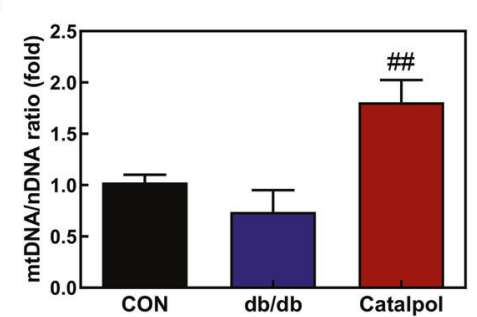

g

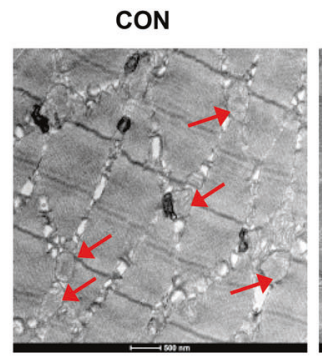

$\mathrm{db} / \mathbf{d b}$
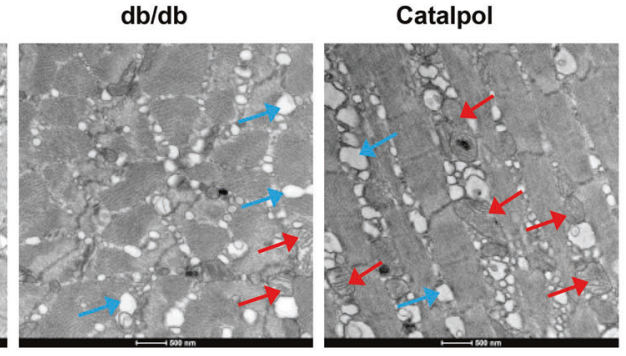

h

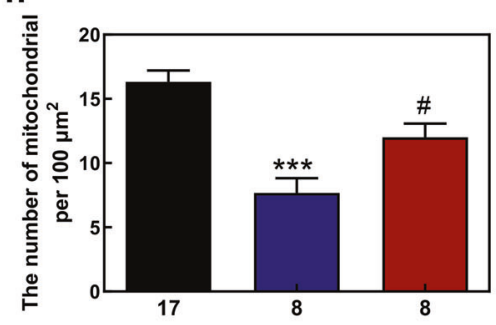

i

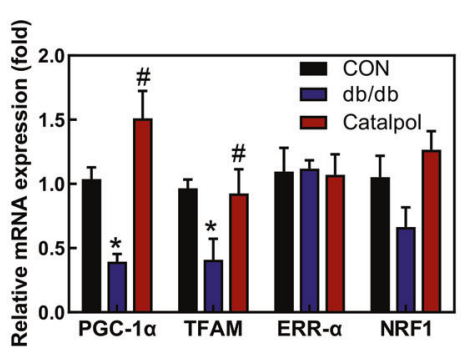

j

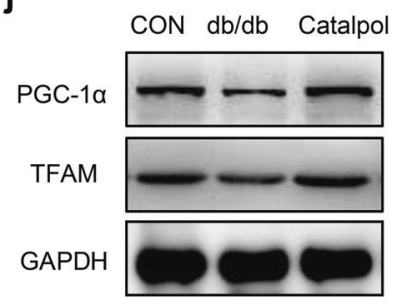

k

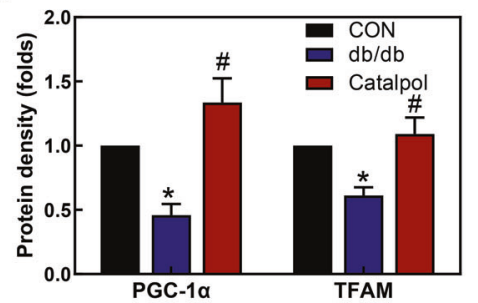

Fig. 1 Catalpol increases mitochondrial biogenesis in $\boldsymbol{d} b / \boldsymbol{d} b$ mice. $\mathbf{a}$ The insulin tolerance results and $\mathbf{b}$ the areas under the curves (AUCs) for the insulin tolerance test are shown. c The glucose tolerance results from the glucose tolerance test are shown. $\mathbf{d}$ Mitochondrial membrane potential, e ATP production, and f mtDNA copy number were determined in the TA muscle of $d b / d b$ mice. g Representative electron micrographs of mitochondria in the TA muscle. Red arrowheads: mitochondria; blue arrowheads: lipid droplets. Scale bar, $500 \mathrm{~nm}$. $\mathbf{h}$ The number of mitochondria calculated using Innerview 2.0 software (Lumenera). $\mathbf{i}$ The relative mRNA levels of PGC-1 $\alpha$, TFAM, ERR- $\alpha$, and NRF1 measured by RT-qPCR. GAPDH served as the control housekeeping gene. j, $\mathbf{k}$ PGC-1 $\alpha$ and TFAM protein levels in mouse TA muscle measured by Western blot analysis. The data are expressed as the means $\pm \mathrm{SD}(n=8$ animals $),{ }^{*} P<0.05,{ }^{* *} P<0.001$ vs. control mice; ${ }^{\#} P<0.05,{ }^{\# \#} P<0.01$ vs. $d b / d b$ mice.

whereas the proportion of MHC II fibers was significantly increased in $d b / d b$ mice compared with control mice (Fig. 2a). However, the proportions of MHC I and II fibers did not differ between the catalpol treatment and control groups (Fig. 2b, c). Catalpol increased the proportion of $\mathrm{MHC}$ I fibers and decreased that of MHC II fibers in $d b / d b$ mice. In addition, the p-AMPK/tAMPK and $\mathrm{p}-\mathrm{ACC} / \mathrm{t}-\mathrm{ACC}$ protein ratios were notably increased in $d b / d b$ mice after catalpol treatment. (Fig. 2d, e). To further demonstrate that catalpol could cause a switch from glycolysis back to oxidative phosphorylation in $d b / d b$ mice, we examined these two types of metabolism in the TA muscle. Regarding glycolysis, the protein level of PFKP (Fig. 2f, g) and the lactate level (Fig. $2 \mathrm{~h}$ ) in the TA muscle did not differ between the $d b / d b$ group and the control group. To examine oxidative phosphorylation, we detected the protein expression of the OXPHOS subunits, including NDUFS1 (Complex I), SDHA (Complex II), UQCRC1
(Complex III), COX1 (Complex IV), and ATP5A1 (Complex V). The levels of these proteins were markedly decreased in the TA muscle of $d b / d b$ mice, but these decreases were reversed by catalpol (Fig. 2i, j).

Catalpol increases mitochondrial biogenesis in C2C12 myotubes To further examine the effects of catalpol on mitochondrial activity, we performed an ATP assay. Catalpol increased ATP production in a dose-dependent manner (Fig. 3a) and markedly increased the mtDNA copy number in $\mathrm{C} 2 \mathrm{C} 12$ myotubes (Fig. $3 \mathrm{~b}$ ). Thus, catalpol may increase mitochondrial biogenesis in skeletal muscle. Moreover, consistent with the in vivo results (Fig. $2 \mathrm{~d}, \mathrm{e}$ ), catalpol significantly increased the $\mathrm{p}-\mathrm{AMPK} / \mathrm{t}-\mathrm{AMPK}$ and $\mathrm{p}-\mathrm{ACC} / \mathrm{t}-$ ACC protein ratios in $\mathrm{C} 2 \mathrm{C} 12$ myotubes (Fig. 3c). PGC-1a and TFAM protein levels were also increased after catalpol treatment in C2C12 myotubes (Fig. 3d). 
a

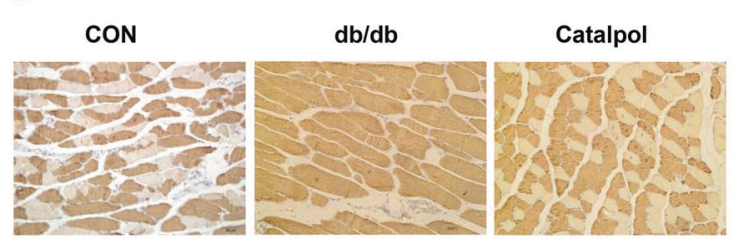

b

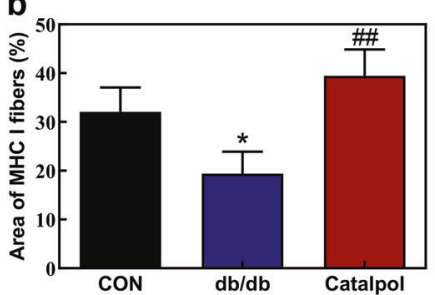

C

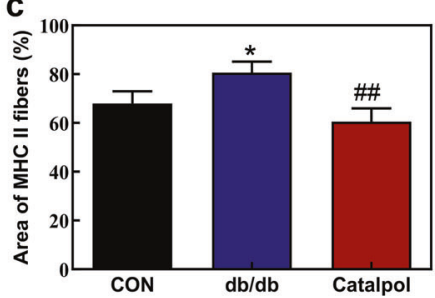

d

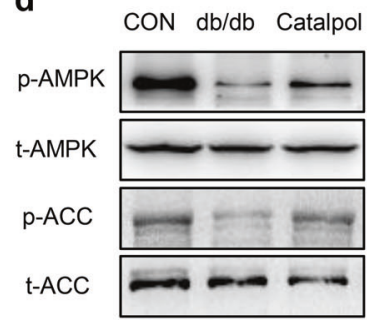

h

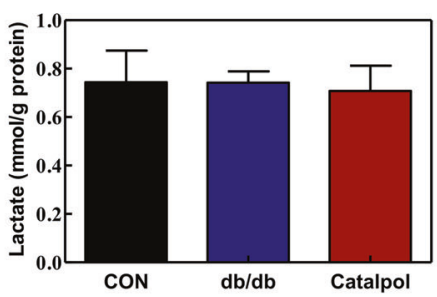

e

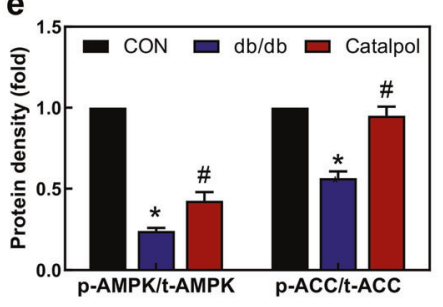

f

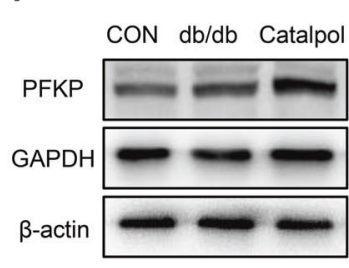

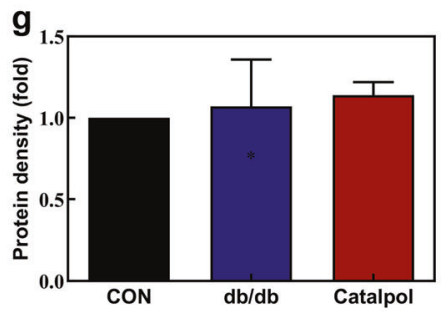

i
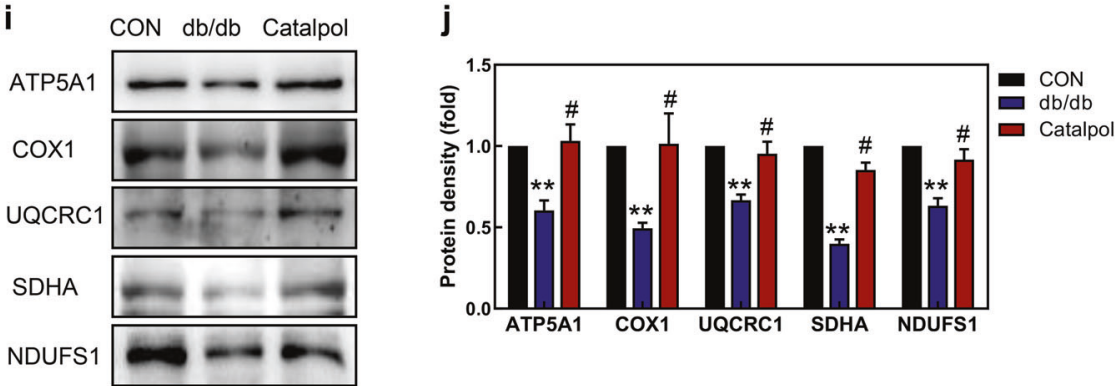

Fig. 2 Catalpol prevents the transformation of muscle fiber types in $\mathbf{d b} / \mathbf{d} \boldsymbol{b}$ mice. a TA muscles stained with an anti-MHC II antibody and visualized under a light microscope (magnification $200 \times$ ). The percentages of b MHC I-positive and $\mathbf{c}$ MHC II-positive areas calculated using Innerview 2.0 software. d, e Western blot analysis using anti-phospho-Thr172-AMPK, anti-AMPK, anti-phospho-Ser79-Acetyl-CoA (ACC) and anti-ACC antibodies to measure protein levels in the TA muscle of $d b / d b$ mice. $\mathbf{f}, \mathbf{g}$ PFKP protein levels in the TA muscle. $\mathbf{h}$ lactate levels in the TA muscle. $\mathbf{i}$, $\mathbf{j}$ The protein levels of NDUFS1 (Complex I), SDHA (Complex II), UQCRC1 (Complex III), COX1 (Complex IV), and ATP5A1 (Complex V) measured by Western blot analysis. The data are expressed as the means \pm SD $\left(n=3\right.$ animals), ${ }^{*} P<0.05,{ }^{* * *} P<0.001$ vs. control mice; ${ }^{\#} P<$ $0.05,{ }^{\# \#} P<0.01$ vs. $d b / d b$ mice.

Catalpol ameliorates the mitochondrial dysfunction induced by high glucose levels in $\mathrm{C} 2 \mathrm{C} 12$ myotubes

Although catalpol treatment increased mitochondrial biogenesis in $\mathrm{C} 2 \mathrm{C} 12$ myotubes, whether catalpol protects $\mathrm{C} 2 \mathrm{C} 12$ myotubes against high-glucose (HG)-induced mitochondrial dysfunction remained unknown. We used $50 \mathrm{mM}$ glucose to simulate an HG environment in $\mathrm{C} 2 \mathrm{C} 12$ myotubes. To explore whether catalpol inhibits HG-induced mitochondrial fragmentation, we used Mito-Tracker Red staining to monitor mitochondrial morphology. In C2C12 myotubes cultured with a normal level of glucose $(1000 \mathrm{mg} / \mathrm{L})$, the mitochondria were intact and elongated. In contrast, the mitochondria were shorter and fragmented following HG treatment (Fig. 4a). The p-AMPK/t-AMPK and p-ACC/t-ACC protein ratios were significantly decreased in HG-treated $\mathrm{C} 2 \mathrm{C} 12$ myotubes, and this effect was reversed by catalpol or metformin treatment (Fig. 4b). Additionally, catalpol significantly increased the protein levels of PGC-1a and TFAM compared with their levels in the HG group (Fig. 4C).

The hypoglycemic effect of catalpol is decreased by AMPK inhibitors

Prior studies have suggested that AMPK is important for the maintenance of mitochondrial function $[34,35]$. We found that the p-AMPK/t-AMPK protein ratio increased notably after catalpol treatment both in vivo (Fig. $2 \mathrm{~d}$, e) and in vitro (Fig. $3 \mathrm{c}$ ). To explore the role of AMPK in the hypoglycemic effect of catalpol, compound $\mathrm{C}$, an AMPK inhibitor, was used to block AMPK phosphorylation in $\mathrm{C} 2 \mathrm{C} 12$ myotubes (Fig. 5a). As expected, both glucose consumption and ATP production decreased following the inhibition of AMPK-mediated phosphorylation. Both catalpol and metformin increased glucose consumption and ATP production via the activation of AMPK signaling (Fig. $5 \mathrm{c}$ ). Notably, the catalpol-induced increases in PGC-1a and TFAM protein levels decreased after AMPK inhibition (Fig. 5b). Strict regulation of AMPK phosphorylation is essential for optimal mitochondrial function [36]. Thus, AMPK may play an important role in the hypoglycemic effect of catalpol.

\section{DISCUSSION}

Previous studies have shown that catalpol effectively lowers fasting blood glucose levels in rodent models of type $1 \mathrm{DM}$ and T2DM $[25,37]$. Consistent with these works, we also found that catalpol significantly reduced blood glucose levels in $d b / d b$ mice. Moreover, we identified a novel molecular mechanism by which catalpol lowers blood glucose levels. Using $d b / d b$ mice and $\mathrm{C} 2 \mathrm{C} 12$ cells, we showed that catalpol increases mitochondrial biogenesis by activating the AMPK/PGC-1a/TFAM signaling pathway, which improves both mitochondrial function and glucose homeostasis in skeletal muscle (Fig. 6). Overall, AMPK/PGC-1a/TFAM signaling plays a vital role in the hypoglycemic effect of catalpol.

Mitochondrial dysfunction and insulin resistance are closely related; insulin resistance can trigger mitochondrial dysfunction in the liver and skeletal muscle [38], and mitochondrial injury can develop after insulin resistance is established [39]. Therefore, increased mitochondrial function is critical for improving insulin 


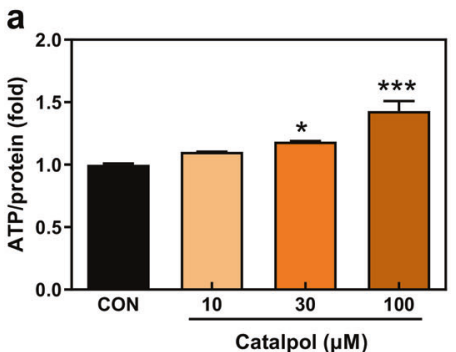

C

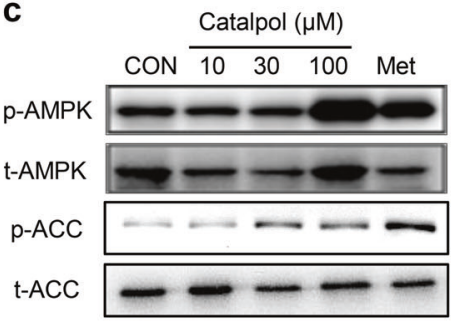

b
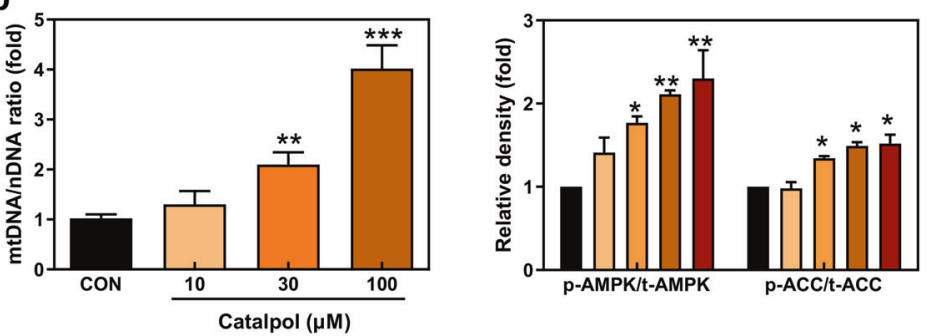

d
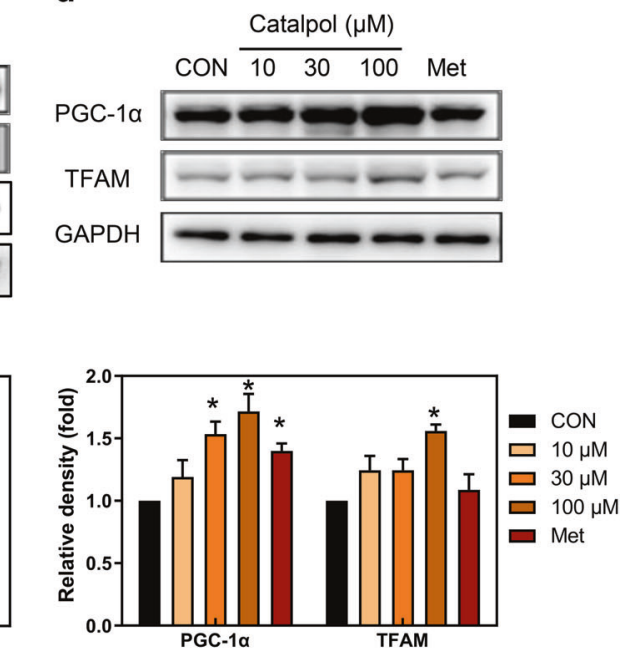

Fig. 3 Catalpol increases mitochondrial biogenesis in C2C12 myotubes. a ATP production and $\mathbf{b}$ the mtDNA copy number were measured in $\mathrm{C} 2 \mathrm{C} 12$ myotubes exposed to catalpol for $24 \mathrm{~h}$. c Western blot analysis using anti-phospho-Thr172-AMPK, anti-AMPK, anti-phospho-Ser79ACC and anti-ACC antibodies to determine protein levels and the ratios of $\mathrm{p}-\mathrm{AMPK} / \mathrm{t}-\mathrm{AMPK}$ and $\mathrm{p}-\mathrm{ACC} \mathrm{t}-\mathrm{ACC}$. $\mathbf{d}$ Western blot analysis of PGC$1 \alpha$ and TFAM (CON: control group; Met: metformin $1 \mathrm{mM}$ ). Values are expressed as the means $\pm \mathrm{SD}(n=3),{ }^{*} P<0.05,{ }^{* *} P<0.01,{ }^{* * *} P<0.001$ vs. control.

a

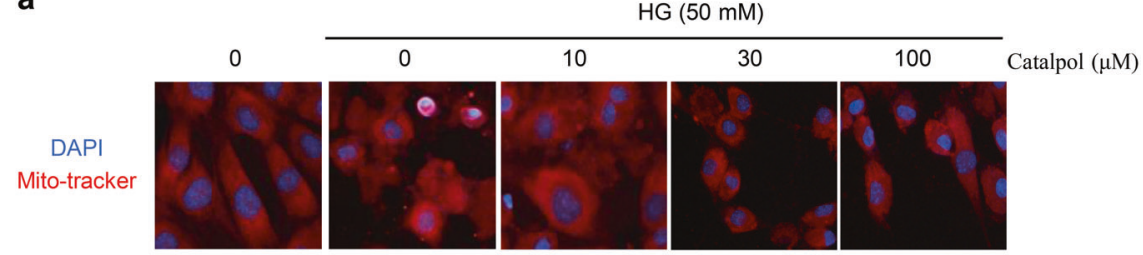

b

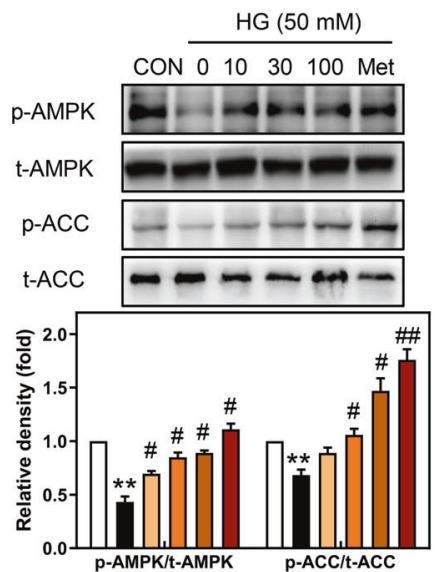

C $\operatorname{coN} \frac{\mathrm{HG}(50 \mathrm{mM})}{01030100 \mathrm{Met}}$
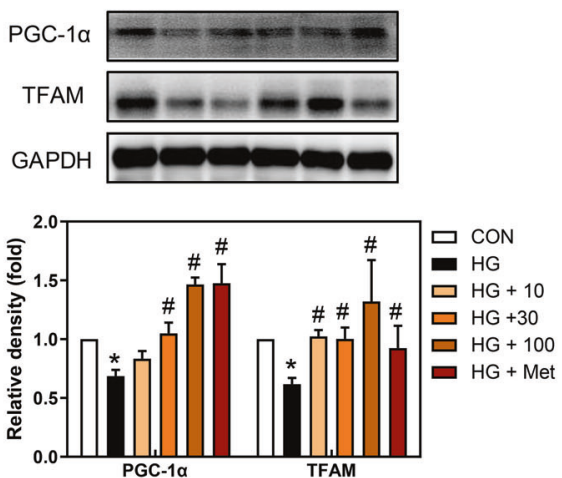

Fig. 4 Catalpol protects against HG-induced mitochondrial dysfunction. a C2C12 myotubes stained with Mito-Tracker (red) and visualized by fluorescence microscopy after exposure to HG $(50 \mathrm{mM})$, as described in the Methods (magnification, $\times 400)$. Cell nuclei were stained with DAPI (blue). b Western blot analysis using anti-phospho-Thr172-AMPK, anti-AMPK, anti-phospho-Ser79-ACC and anti-ACC antibodies to determine protein levels and the ratios of p-AMPK/t-AMPK and p-ACC/t-ACC. c Western blot analysis of PGC-1 $\alpha$ and TFAM (CON: control group; Met: metformin $1 \mathrm{mM})$. Values are expressed as the means $\pm \mathrm{SD}(n=3),{ }^{*} P<0.05,{ }^{* *} P<0.01$ vs. control; ${ }^{\#} P<0.05,{ }^{\# \#} P<0.01$ vs. HG.

resistance. We found that mitochondrial function was impaired in the skeletal muscle of $d b / d b$ mice. After catalpol treatment, mitochondrial biogenesis was markedly increased. Catalpol also ameliorated mitochondrial dysfunction induced by HG in skeletal muscle cells. Together, the results show that catalpol increased mitochondrial biogenesis, thereby ameliorating mitochondrial impairment in skeletal muscle. Thus, catalpol increased glucose uptake and ATP production by skeletal muscle, reducing the blood glucose level.

It is generally acknowledged that AMPK, a sensor of cellular energy status, serves as a key regulator of glucose uptake, fatty acid oxidation, and mitochondrial biogenesis [40]. AMPK is a well-known upstream regulator of PGC-1a expression in skeletal muscle. AMPK activation increases the expression and phosphorylation of PGC-1a, 
a
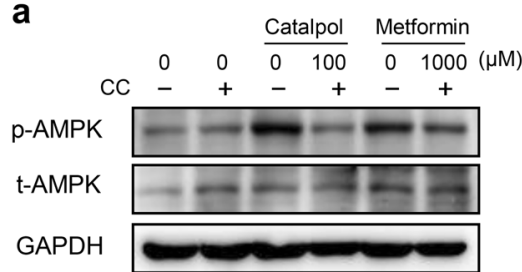

C

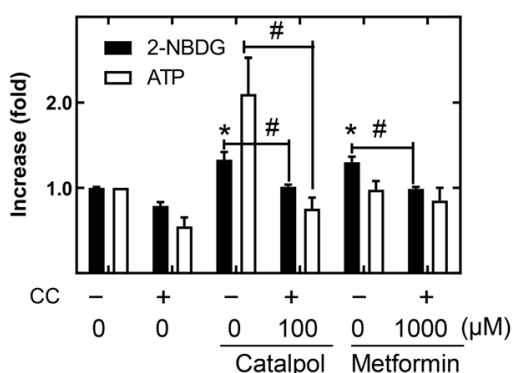

b
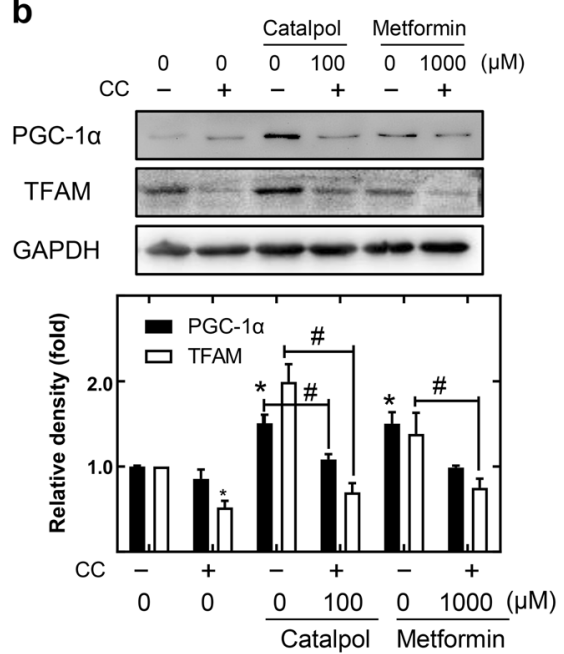

Fig. 5 The hypoglycemic effect of catalpol is achieved by increased AMPK-mediated mitochondrial biogenesis. Differentiated myotubes were incubated in serum-free media with $2 \mu \mathrm{M}$ compound $\mathrm{C}$ for $2 \mathrm{~h}$ and then in fresh serum-free media with 10,30, or $100 \mu \mathrm{M}$ catalpol, or with $1 \mathrm{mM}$ metformin, for $24 \mathrm{~h}$. a Protein levels of p-AMPK (upper panel) and t-AMPK (lower panel). b Western blot analysis of PGC-1 $\alpha$ and TFAM in C2C12 myotubes. c Effect of catalpol on 2-NBDG uptake and ATP production by C2C12 myotubes. Values are expressed as the means \pm SD $(n=3),{ }^{*} P<0.05$ vs. control. ${ }^{\#} P<0.05$.

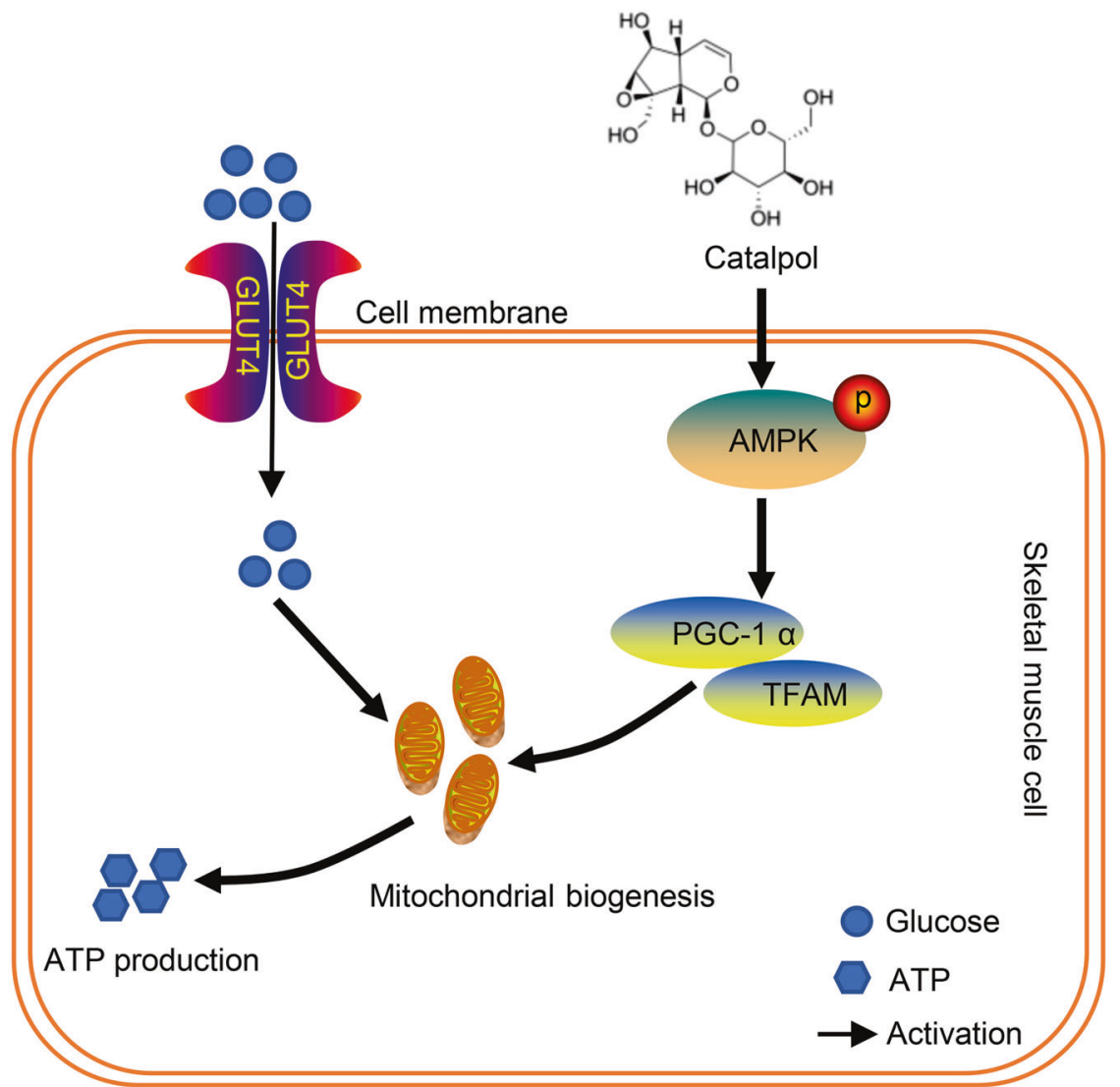

Fig. 6 Hypoglycemic mechanism of catalpol in $\boldsymbol{d} b / \boldsymbol{d} b$ mouse skeletal muscle. Catalpol increased AMPK/PGC-1 $\alpha / T F A M-m e d i a t e d$ mitochondrial biogenesis in skeletal muscle cells. Increased mitochondrial biogenesis resulted in increased glucose uptake and ATP production in skeletal muscle cells.

further stimulating mitochondrial biogenesis [41]. As mentioned above, the activation of mitochondrial biogenesis may be useful to treat T2DM and/or insulin resistance. Previous studies have suggested that AMPK stimulates mitochondrial biogenesis and $\beta$ oxidation via PGC-1a upregulation in skeletal muscle $[34,42]$.
Upregulated expression of AMPK and PGC-1a increased mitochondrial function and muscle glucose metabolism, as shown using both glucose uptake and ATP assays, suggesting a strong association between AMPK activity and the hypoglycemic effects of catalpol. Additionally, the hypoglycemic effect was reduced when catalpol 
was coadministered with compound C (an AMPK inhibitor). Thus, AMPK-mediated mitochondrial biogenesis may be crucial for the hypoglycemic effect of catalpol.

The regulation of mitochondrial replication and biogenesis increases mitochondrial function. Mitochondria play vital roles in diverse metabolic pathways $[12,43,44]$. PGC-1a stimulates oxidative metabolism and increases the number of mitochondria. PGC-1a is strongly expressed in the skeletal muscle, heart, white adipose tissue, and brain, all of which are highly oxidative tissues [45]. We found that $d b / d b$ mice exhibited decreased PGC-1 $a$ and TFAM levels in skeletal muscle, consequently, reductions in mitochondrial biogenesis and the number of mitochondria. Additionally, $d b / d b$ mice exhibited a lower mitochondrial membrane potential and ATP production in skeletal muscle. Our results are consistent with earlier data showing mitochondrial dysfunction and reduced mitochondrial biogenesis in the skeletal muscles of $d b / d b$ mice. Catalpol increased the protein levels of both PGC-1a and TFAM in the skeletal muscle of $d b / d b$ mice and $\mathrm{C} 2 \mathrm{C} 12$ myotubes, consistent with the observed increases in mtDNA copy number. We found that PGC-1a/TFAM-mediated mitochondrial biogenesis may play a vital role in the hypoglycemic effect of catalpol.

In summary, mitochondrial dysfunction was evident in the skeletal muscle of $d b / d b$ mice. The catalpol-induced activation of AMPK/PGC-1a/TFAM signaling increased mitochondrial biogenesis in skeletal muscle, thereby increasing glucose uptake and ATP production. Hence, skeletal muscle mitochondria may be a useful target for the treatment of T2DM.

\section{ACKNOWLEDGEMENTS}

This work was supported by grants from the National Natural Science Foundation of China (No. 81403154, 81773827, 81573514, 81773995, 81873084, 81573690); the Scholar of the 14th Batch of "Six Talents Peak" High-level Talent Selection program (SWYY-094); the Postgraduate Research Practice Innovation Program of Jiangsu Province (KYCX19-0763); the "Double First-Class" University project (CPU2018GY33); the Qinghai Province High-End Innovative Thousand Talents Program; and the Tianshan Cedar Project of the Xinjiang Uygur Autonomous Region (2018XS21)

\section{AUTHOR CONTRIBUTIONS}

DQX and LW analyzed the data, contributed to discussions and wrote the manuscript. DQX, CJL, and SSF collected the data. ZJL and HFH analyzed the data. LXS reviewed the manuscript. TW and ZZJ conceived the experiments, analyzed the data and edited/reviewed the manuscript. LYZ is the guarantor of this work, had full access to all the data in the study and takes responsibility for the integrity of the data and the accuracy of the data analysis.

\section{ADDITIONAL INFORMATION}

Conflict of interest: The authors declare that they have no conflict of interest.

\section{REFERENCES}

1. American Diabetes A. Diagnosis and classification of diabetes mellitus. Diabetes Care. 2014;37:S81-90.

2. Oriente F, lovino S, Cabaro S, Cassese A, Longobardi E, Miele C, et al. Prep1 controls insulin glucoregulatory function in liver by transcriptional targeting of SHP1 tyrosine phosphatase. Diabetes. 2011;60:138-47.

3. Zamora M, Pardo R, Villena JA. Pharmacological induction of mitochondrial biogenesis as a therapeutic strategy for the treatment of type 2 diabetes. Biochem Pharmacol. 2015;98:16-28.

4. Yaribeygi $H$, Atkin SL, Sahebkar A. Mitochondrial dysfunction in diabetes and the regulatory roles of antidiabetic agents on the mitochondrial function. J Cell Physiol. 2019;234:8402-10.

5. Rector RS, Thyfault JP, Uptergrove GM, Morris EM, Naples SP, Borengasser SJ, et al. Mitochondrial dysfunction precedes insulin resistance and hepatic steatosis and contributes to the natural history of non-alcoholic fatty liver disease in an obese rodent model. J Hepatol. 2010;52:727-36.

6. Hesselink MKC, Schrauwen-Hinderling V, Schrauwen P. Skeletal muscle mitochondria as a target to prevent or treat type 2 diabetes mellitus. Nat Rev Endocrinol. 2016;12:633-45.
7. Deng XH, Liu JJ, Sun XJ, Dong JC, Huang JH. Benzoylaconine induces mitochondrial biogenesis in mice via activating AMPK signaling cascade. Acta Pharmacol Sin. 2019;40:658-65

8. Hancock ML, Meyer RC, Mistry M, Khetani RS, Wagschal A, Shin T, et al. Insulin receptor associates with promoters genome-wide and regulates gene expression. Cell. 2019;177:722-36.

9. Schrauwen-Hinderling VB, Kooi ME, Hesselink MK, Jeneson JA, Backes WH, van Echteld $\mathrm{CJ}$, et al. Impaired in vivo mitochondrial function but similar intramyocellular lipid content in patients with type 2 diabetes mellitus and BMI-matched control subjects. Diabetologia. 2007;50:113-20.

10. Xu DQ, Jiang ZZ, Sun ZR, Wang L, Zhao GL, Hassan HM, et al. Mitochondrial dysfunction and inhibition of myoblast differentiation in mice with high-fat-dietinduced pre-diabetes. J Cell Physiol. 2019;234:7510-23.

11. Barreiro E, Puig-Vilanova E, Marin-Corral J, Chacon-Cabrera A, Salazar-Degracia A, Mateu $X$, et al. Therapeutic approaches in mitochondrial dysfunction, proteolysis, and structural alterations of diaphragm and gastrocnemius in rats with chronic heart failure. J Cell Physiol. 2016;231:1495-513.

12. Andreux PA, Houtkooper RH, Auwerx J. Pharmacological approaches to restore mitochondrial function. Nat Rev Drug Discov. 2013;12:465-83.

13. Befroy DE, Petersen KF, Dufour S, Mason GF, de Graaf RA, Rothman DL, et al. Impaired mitochondrial substrate oxidation in muscle of insulin-resistant offspring of type 2 diabetic patients. Diabetes. 2007;56:1376-81.

14. Ritov VB, Menshikova EV, He J, Ferrell RE, Goodpaster BH, Kelley DE. Deficiency of subsarcolemmal mitochondria in obesity and type 2 diabetes. Diabetes. 2005;54:8-14.

15. Fang WJ, Wang CJ, He Y, Zhou YL, Peng XD, Liu SK. Resveratrol alleviates diabetic cardiomyopathy in rats by improving mitochondrial function through PGC1a deacetylation. Acta Pharmacol Sin. 2018;39:59-73.

16. Yan WJ, Zhang HF, Liu PL, Wang H, Liu JY, Gao C, et al. Impaired mitochondrial biogenesis due to dysfunctional adiponectin-AMPK-PGC-1 a signaling contributing to increased vulnerability in diabetic heart. Basic Res Cardiol. 2013;108:329-43.

17. Whitaker RM, Corum D, Beeson CC, Schnellmann RG. Mitochondrial biogenesis as a pharmacological target: a new approach to acute and chronic diseases. Annu Rev Pharm Toxicol. 2016;56:229-49.

18. Villena JA. New insights into PGC-1 coactivators: redefining their role in the regulation of mitochondrial function and beyond. FEBS J. 2015;282:647-72.

19. Gleyzer N, Vercauteren K, Scarpulla RC. Control of mitochondrial transcription specificity factors (TFB1M and TFB2M) by nuclear respiratory factors (NRF-1 and NRF-2) and PGC-1 family coactivators. Mol Cell Biol. 2005;25:1354-66.

20. Yun CW, Han YS, Lee SH. PGC-1a controls mitochondrial biogenesis in drugresistant colorectal cancer cells by regulating endoplasmic reticulum stress. Int J Mol Sci. 2019;20:1707-20.

21. Halling JF, Jessen $\mathrm{H}$, Nohr-Meldgaard J, Thiellesen Buch B, Masselkhi Christensen $\mathrm{N}$, Gudiksen A, et al. PGC-1a regulates mitochondrial properties beyond biogenesis with aging and exercise training. Am J Physiol Endocrinol Metab. 2019;317:E513-25

22. Xu DQ, Wang L, Jiang ZZ, Zhao GL, Hassan HM, Sun LX, et al. A new hypoglycemic mechanism of catalpol revealed by enhancing MyoD/MyoG-mediated myogenesis. Life Sci. 2018;209:313-23.

23. Dong Z, Chen CX. Effect of catalpol on diabetic nephropathy in rats. Phytomedicine. 2013;20:1023-9.

24. Zhou J, Xu G, Ma S, Li F, Yuan M, Xu HB, et al. Catalpol ameliorates high-fat dietinduced insulin resistance and adipose tissue inflammation by suppressing the JNK and NF-kappaB pathways. Biochem Biophys Res Commun. 2015;467:853-8.

25. Li X, Xu ZM, Jiang ZZ, Sun LX, Ji JZ, Miao JS, et al. Hypoglycemic effect of catalpol on high-fat diet/streptozotocin-induced diabetic mice by increasing skeletal muscle mitochondrial biogenesis. Acta Biochim Biophys Sin (Shanghai). 2014;46:738-48.

26. Xu ZM, Zhang LY, Li XJ, Jiang ZZ, Sun LX, Zhao GL, et al. Mitochondrial fusion/ fission process involved in the improvement of catalpol on high glucose-induced hepatic mitochondrial dysfunction. Acta Biochim Biophys Sin (Shanghai). 2015;47:730-40.

27. Yi JS, Park JS, Ham YM, Nguyen N, Lee NR, Hong J, et al. MG53-induced IRS-1 ubiquitination negatively regulates skeletal myogenesis and insulin signalling. Nat Commun 2013:4:2354-77.

28. Park SY, Kim MH, Ahn JH, Lee SJ, Lee JH, Eum WS, et al. The stimulatory effect of essential fatty acids on glucose uptake involves both Akt and AMPK activation in C2C12 skeletal muscle cells. Korean J Physiol Pharmacol. 2014;18:255-61.

29. Zhao GL, Xu DQ, Yuan ZQ, Jiang ZZ, Zhou W, Li ZJ, et al. 8-Methoxypsoralen disrupts MDR3-mediated phospholipids efflux and bile acid homeostasis and its relevance to hepatotoxicity. Toxicology 2017;386:40-8.

30. Kim JY, Choi MJ, So B, Kim HJ, Seong JK, Song W. The preventive effects of 8 weeks of resistance training on glucose tolerance and muscle fiber type composition in zucker rats. Diabetes Metab J. 2015:39:424-33. 
31. Houstek J, Pickova A, Vojtiskova A, Mracek T, Pecina P, Jesina P. Mitochondrial diseases and genetic defects of ATP synthase. Biochim Biophys Acta. 2006;1757:1400-5.

32. Chen XL, Wei SS, Yang FQ. Mitochondria in the pathogenesis of diabetes: a proteomic view. Protein Cell. 2012;3:648-60.

33. Venojarvi M, Puhke $\mathrm{R}$, Hamalainen $\mathrm{H}$, Marniemi J, Rastas $\mathrm{M}$, Rusko $\mathrm{H}$, et al. Role of skeletal muscle-fibre type in regulation of glucose metabolism in middle-aged subjects with impaired glucose tolerance during a long-term exercise and dietary intervention. Diabetes Obes Metab. 2005;7:745-54.

34. Jager S, Handschin C, Pierre J, Spiegelman BM. AMP-activated protein kinase (AMPK) action in skeletal muscle via direct phosphorylation of PGC-1a. Proc Nat Acad Sci USA. 2007;104:12017-22.

35. Kabra UD, Pfuhlmann K, Migliorini A, Keipert S, Lamp D, Korsgren O, et al. Direct substrate delivery into mitochondrial fission-deficient pancreatic islets rescues insulin secretion. Diabetes. 2017;66:1247-57.

36. Chopra I, Li HF, Wang H, Webster KA. Phosphorylation of the insulin receptor by AMP-activated protein kinase (AMPK) promotes ligand-independent activation of the insulin signalling pathway in rodent muscle. Diabetologia 2012;55:783-94.

37. Bao QW, Shen XZ, Qian L, Gong C, Nie MX, Dong Y. Anti-diabetic activities of catalpol in $d b / d b$ mice. Korean J Physiol Pharmacol. 2016;20:153-60.
38. Affourtit C. Mitochondrial involvement in skeletal muscle insulin resistance: a case of imbalanced bioenergetics. Biochim Biophys Acta. 2016;1857:1678-93.

39. Petersen KF, Befroy D, Dufour S, Dziura J, Ariyan C, Rothman DL, et al. Mitochondrial dysfunction in the elderly: possible role in insulin resistance. Science. 2003;300:1140-2.

40. Carling D. AMPK signalling in health and disease. Curr Opin Cell Biol. 2017;45:31-7.

41. Marin TL, Gongol B, Zhang F, Martin M, Johnson DA, Xiao H, et al. AMPK promotes mitochondrial biogenesis and function by phosphorylating the epigenetic factors DNMT1, RBBP7, and HAT1. Sci Signal. 2017;10:7478-98.

42. Misu H, Takayama H, Saito $Y$, Mita $Y$, Kikuchi A, Ishii $K$, et al. Deficiency of the hepatokine selenoprotein $P$ increases responsiveness to exercise in mice through upregulation of reactive oxygen species and AMP-activated protein kinase in muscle. Nat Med. 2017;23:508-16.

43. Holloway GP. Mitochondrial function and dysfunction in exercise and insulin resistance. Appl Physiol Nutr Metab. 2009;34:440-6.

44. Wanagat J, Hevener AL. Mitochondrial quality control in insulin resistance and diabetes. Curr Opin Genet Dev. 2016;38:118-26.

45. Wu HJ, Deng XN, Shi YH, Su Y, Wei JY, Duan HJ. PGC-1a, glucose metabolism and type 2 diabetes mellitus. J Endocrinol. 2016;229:R99-115. 